\title{
HYPERSENSITIVITY PNEUMONITIS: ESSENTIAL RADIOLOGIC AND PATHOLOGIC FINDINGS
}

Teri J. Franks, $M D^{a, \star}$, Jeffrey R. Galvin, $M D^{b, c}$

\section{KEYWORDS}

- Hypersensitivity pneumonitis • Hypersensitivity pneumonia • Extrinsic allergic alveolitis

- Non-necrotizing granulomas

\section{ABSTRACT}

H ypersensitivity pneumonitis is a diffuse, granulomatous interstitial lung disease caused by repeated exposure to a wide spectrum of environmental antigens. Clinical, radiologic, and histologic findings are quite variable and mimic many other diseases; therefore, diagnosis depends on a constellation of findings rather than a single defining feature. High-resolution computed tomography (HRCT) plays a critical role in this setting. The presence of centrilobular nodules, multifocal ground glass opacities, and evidence of air trapping in the expiratory phase of respiration is highly suggestive of hypersensitivity pneumonitis and can direct clinicians to the correct diagnosis. For the pathologist, typical HRCT findings not only corroborate the diagnosis of hypersensitivity pneumonitis when the characteristic triad of airway-centered chronic interstitial inflammation, interstitial poorly formed non-necrotizing granulomas, and organizing pneumonia is present on biopsy, but also improve diagnostic confidence in biopsies lacking components of the diagnostic histologic triad. Importantly, the presence of focal or unilateral imaging findings should prompt a careful search by the pathologist for an infectious etiology, thus avoiding errors in determining the significance of granulomas on biopsy. This article reviews the essential radiologic and pathologic findings in hypersensitivity pneumonitis, discusses the primary considerations in the differential diagnosis, and offers an approach to biopsy evaluation.

\section{OVERVIEW}

Hypersensitivity pneumonitis, also known as extrinsic allergic alveolitis, is an interstitial lung disease caused by an exaggerated immunologic response to inhalation of aerosolized antigenic particles found in a wide variety of occupational, domestic, and recreational environments. Although the number of antigens known to cause hypersensitivity pneumonitis is extensive and ever increasing, they can be organized into three groups: microbial agents, animal/insect proteins, and low-molecular weight chemicals (Table 1). Among these, microbial agents, in particular those that give rise to farmer's lung; pigeon breeder's/ bird fancier's disease; and various exposures to fungi are most common. ${ }^{1}$

Typically, hypersensitivity pneumonitis is classified as acute, subacute, or chronic; however,

\footnotetext{
a Department of Pulmonary \& Mediastinal Pathology, Armed Forces Institute of Pathology, 6825 16th Street NW, Washington, DC 20306, USA

b Department of Radiologic Pathology, Armed Forces Institute of Pathology, 6825 16th Street NW, Washington, DC 20306, USA

c Department of Diagnostic Radiology, and Department of Internal Medicine, Division of Pulmonary/Critical Care Medicine, University of Maryland School of Medicine, 655 West Baltimore Street, Baltimore, MD 21201, USA

* Corresponding author.

E-mail address: tfranks1@mac.com
} 


\begin{tabular}{|c|}
\hline $\begin{array}{c}\text { Key Features } \\
\text { Hypersensitivity PNeUmonitis }\end{array}$ \\
\hline High-resolution computed tomography \\
\hline $\begin{array}{l}\text { Active and residual disease shows diffuse } \\
\text { distribution of: }\end{array}$ \\
\hline - Centrilobular nodules \\
\hline - Multifocal ground glass opacities \\
\hline - Air trapping on expiratory phase studies \\
\hline $\begin{array}{l}\text { In addition, residual disease shows upper and } \\
\text { middle lung zone: }\end{array}$ \\
\hline - Reticular opacities \\
\hline - Honeycombing \\
\hline - Traction bronchiectasis \\
\hline Histology \\
\hline $\begin{array}{l}\text { Active disease shows airway-centered } \\
\text { distribution of: }\end{array}$ \\
\hline - Chronic interstitial inflammation \\
\hline $\begin{array}{l}\text { - Non-necrotizing, poorly circumscribed } \\
\text { granulomas }\end{array}$ \\
\hline - Organizing pneumonia \\
\hline $\begin{array}{l}\text { Residual disease is evidenced by fibrosis in } \\
\text { the histologic patterns of: }\end{array}$ \\
\hline - Nonspecific interstitial pneumonia (NSIP) \\
\hline - Usual interstitial pneumonia (UIP) \\
\hline
\end{tabular}

significant overlap between these clinical presentations occurs. Lacasse and colleagues ${ }^{1}$ set out to determine whether this three-group classification truly reflects categories of patients with distinct clinical features. Cluster analysis of 168 patients identified two rather than three clinical patterns, from which the authors concluded that their results do not support the acute, subacute and chronic classification, and that the subacute category is particularly difficult to define and likely does not represent a separate entity. Acknowledging the need for validation, they suggested reclassification into two groups based on disease activity; active versus residual disease-with the latter representing late emphysematous or fibrotic sequelae-may better reflect distinct clinical categories.

Hypersensitivity pneumonitis is a complex, variable disorder with clinical and laboratory findings that overlap many other diseases., ${ }^{2,3}$ Diagnosis can be difficult and rests on identification of a constellation of clinical, radiologic, and pathologic findings. Consequently, multiple recommendations for diagnostic criteria have been published. ${ }^{3-6}$ None, however, have been validated, raising the question of their diagnostic accuracy. ${ }^{2,7}$ Nonetheless, for the diagnosis of hypersensitivity pneumonitis to be considered, the recommendation of Schulyer ${ }^{3}$ suggests the following should be present:

1. Symptoms compatible with hypersensitivity pneumonitis

2. Evidence of exposure to appropriate antigen by history or detection of serum and/or bronchoalveolar lavage (BAL) fluid antibody

3. Findings compatible with hypersensitivity pneumonitis on chest radiograph or high-resolution computed tomography (HRCT)

4. BAL fluid lymphocytosis if BAL is performed

5. Histologic lung changes compatible with hypersensitivity pneumonitis if lung biopsy is performed

6. Positive natural challenge, which is reproduction of symptoms and laboratory abnormalities after exposure to the suspected environment.

In a prospective multicenter cohort study of 400 patients, Lacasse and colleagues ${ }^{8}$ identified six significant predictors of active hypersensitivity pneumonitis by regression analyses:

1. Exposure to a known offending antigen

2. Positive precipitating antibodies

3. Recurrent episodes of symptoms

4. Inspiratory crackles

5. Symptoms 4 to 8 hours after exposure

6. Weight loss.

They computed the probability of hypersensitivity pneumonitis for each of the 64 combinations generated by the six predictive factors, and suggested this estimate of the probability of hypersensitivity pneumonitis from noninvasive testing may be useful in guiding clinical practice.

\section{GROSS FEATURES}

Gross specimens of large size are not often encountered in hypersensitivity pneumonitis as the lung most often is examined by BAL, transbronchial biopsy, or surgical lung biopsy. Instead, the authors encourage pathologists to use radiologic studies, particularly HRCT, as their in vivo gross lung examination. Radiologic patterns are often characteristic of a specific disease and provide information on type of abnormality and distribution of disease that may not be apparent on the biopsy specimen. ${ }^{9}$ 
Table 1

Etiologic causes and selected examples of hypersensitivity pneumonitis

\begin{tabular}{|c|c|c|}
\hline Antigen & Source & Disease \\
\hline \multicolumn{3}{|l|}{ Microbial agents } \\
\hline \multicolumn{3}{|l|}{ Bacteria } \\
\hline$M$ faeni, $T$ vulgaris & Moldy hay & Farmer's lung \\
\hline T saccharii & Moldy bagasse (pressed sugarcane) & Bagassosis \\
\hline$T$ vulgaris, $T$ candidus & Air conditioners, humidifiers & Air conditioner lung, humidifier lung \\
\hline$B$ cereus & Water & Humidifier lung \\
\hline Mycobacterium avium complex & Mist from hot tubs & Hot tub lung \\
\hline Gram-negative bacteria endotoxins & Cotton, flax, hemp, sisal & Byssinosis \\
\hline \multicolumn{3}{|l|}{ Fungi } \\
\hline C corticale & Moldy maple bark & Maple bark stripper's disease \\
\hline Graphium species, Pullularia species & Moldy wood dust & Sequoiosis \\
\hline T cutaneum, C albidus & Damp wood and mats & Japanese summer house hypersensitivity pneumonitis \\
\hline$P$ frequetans, $A$ fumigatus & Moldy cork dust & Suberosis \\
\hline \multicolumn{3}{|l|}{ Amoebae } \\
\hline N gruberi, A polyhaga, A castellani & Water & Humidifier lung \\
\hline \multicolumn{3}{|l|}{ Animal/insect products } \\
\hline Avian proteins & Bird feathers, droppings & Pigeon breeder's/bird fancier's/poultry worker's lung \\
\hline Aquatic animal proteins & Mollusc shell dust & Mollusc shell hypersensitivity pneumonitis \\
\hline S granarius (wheat weevil) & Dust contaminated grain & Miller's lung, wheat weevil disease \\
\hline \multicolumn{3}{|l|}{ Low-molecular weight chemicals } \\
\hline $\begin{array}{l}\text { Trimellitic anhydride (TMA), Methylene diisocyanate } \\
\text { (MDI), Toluene diisocyanate (TDI), Pyromellitic } \\
\text { dianhydride (PMDA), Naphthylene 1,5 diisocyanate } \\
\text { (NDI) }\end{array}$ & $\begin{array}{l}\text { Plastics, rubber manufacturing, epoxyresin } \\
\text { Polyurethane foam production }\end{array}$ & Chemical worker's lung \\
\hline
\end{tabular}

Abbreviations: A castellani, Acanthamoeba castellani; A fumigatus, Aspergillus fumigatus; A polyhaga, Acanthamoeba polyhaga; B cereus, Bacillus cereus; Calbidus, Cryptococcus

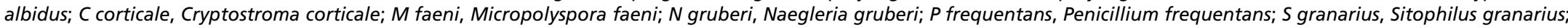
$T$ candidus, Thermoactinomyces candidus; T cutaneum, Trichosporon cutaneum; T saccharii, Thermoactinomyces saccharii; $T$ vulgaris, Thermoactinomyces vulgaris. 

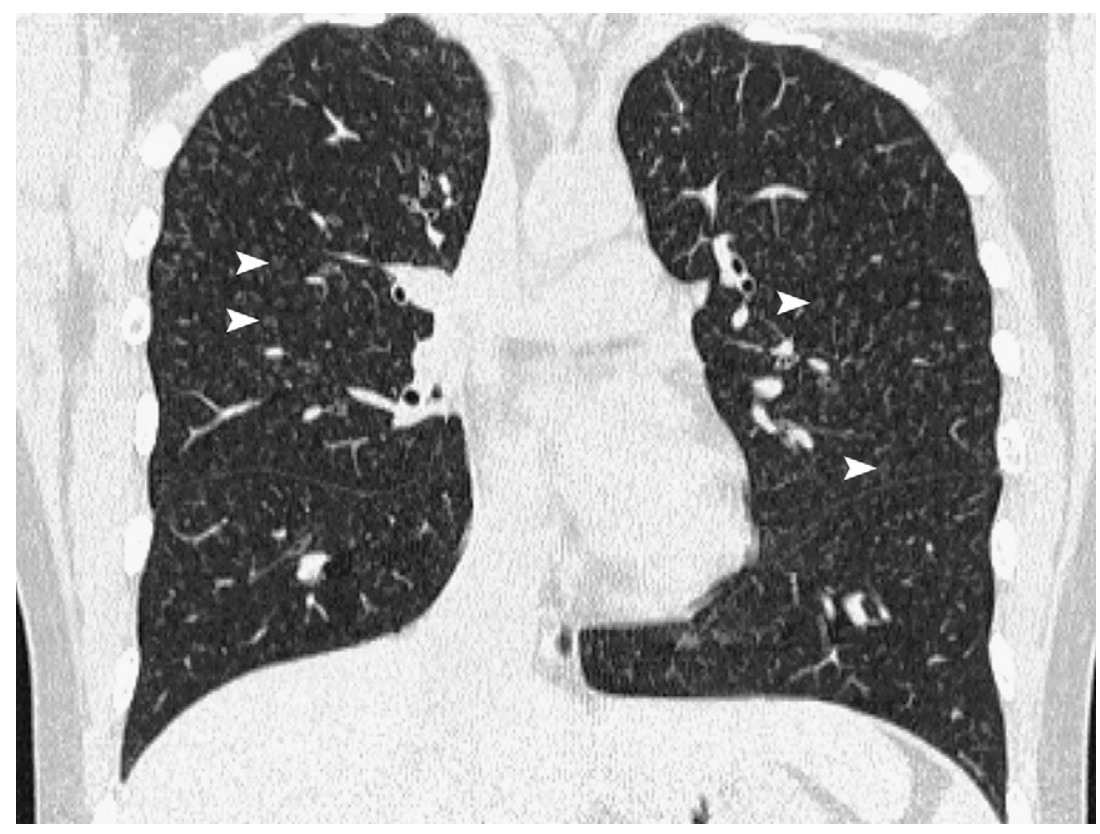

Fig. 1. Hypersensitivity pneumonitis. Coronal reconstruction of highresolution axial computed tomography (CT) data demonstrates centrilobular ground glass nodules (arrowheads) with a uniform distribution from apex to base.
HRCT has replaced the plain chest radiograph as the primary diagnostic imaging modality in patients with hypersensitivity pneumonitis because of its sensitivity and specificity. ${ }^{10}$ Several imaging findings are identified in any phase of hypersensitivity pneumonitis and include: small, indistinct, centrilobular nodules (Fig. 1), multifocal ground glass opacities (Fig. 2), and evidence of air trapping (Fig. 3) in the expiratory phase of respiration. This combination of findings is highly suggestive of hypersensitivity pneumonitis and correlates with functional abnormalities found on pulmonary function tests. ${ }^{11,12}$ The findings, however, are not specific as they also found are in other diseases that demonstrate airway-centered pathology including respiratory bronchiolitis, follicular bronchitis, and asthma.

A reticular pattern, honeycombing (Fig. 4), and traction bronchiectasis (Fig. 5) correlate with fibrosis in the residual phase of hypersensitivity pneumonitis and are associated with reduced survival. ${ }^{13}$ These findings are most severe in the

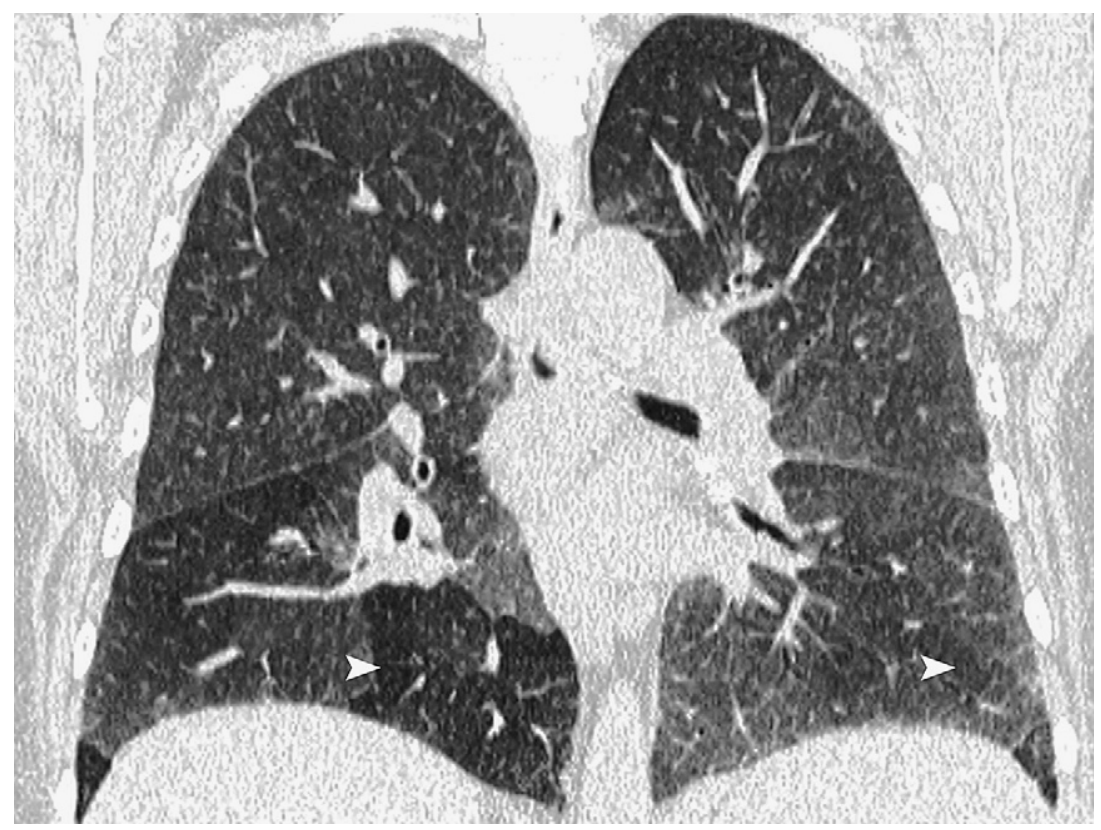

Fig. 2. Hypersensitivity pneumonitis. Coronal reconstruction of high-resolution axial CT data demonstrates widespread areas of ground glass opacification and central airway thickening with focal sparing at the lung bases (arrowheads). 
Fig. 3. Hypersensitivity pneumonitis. Axial CT acquired in the right upper lobe during the inspiratory phase of respiration (left) demonstrates subtle inhomogeneity of density. The image on the right acquired through the same region during the expiratory phase of respiration demonstrates marked accentuation of density differences between secondary pulmonary lobules (arrowheads), consistent with air trapping.

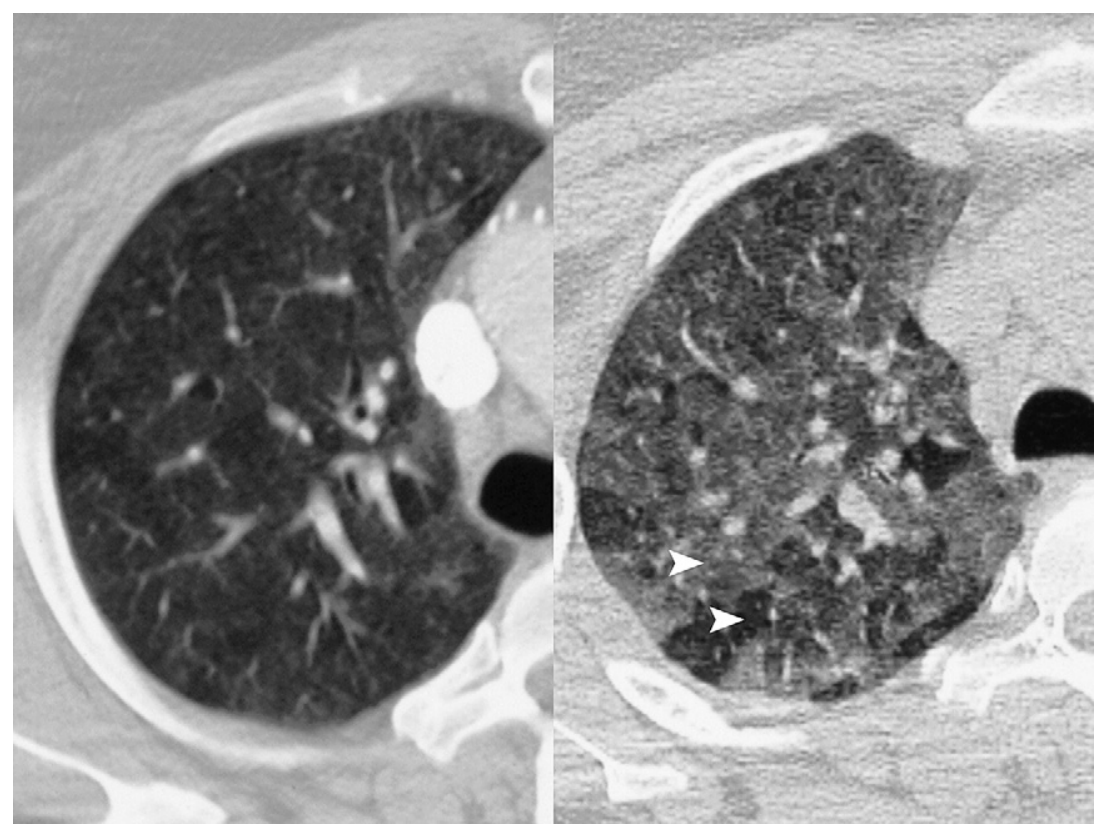

upper and middle lung zones in patients with hypersensitivity pneumonitis. ${ }^{14,15}$ These same findings are also present in idiopathic pulmonary fibrosis (IPF); however, the distribution is different with a strikingly lower lobe predominance (Fig. 6).

The combination of diffuse centrilobular nodules, ground glass opacities and air trapping, and upper and middle lung zone fibrosis is the basis for a probable HRCT diagnosis of hypersensitivity pneumonitis. ${ }^{12,16}$ Imaging with focal or unilateral abnormalities should prompt a careful search for an infectious etiology to explain the patient's symptoms (Figs. 7 and 8).

\section{MICROSCOPIC FEATURES}

Despite the labels hypersensitivity and allergic, hypersensitivity pneumonitis is not an atopic disease and is not associated with increased eosinophils. Surgical lung biopsy generally is required to appreciate the distribution of histologic findings of hypersensitivity pneumonitis since the

Fig. 4. Hypersensitivity pneumonitis. Coronal reconstruction of highresolution axial $\mathrm{CT}$ data demonstrates peripheral reticulation (arrowhead), consolidation (asterisk), and honeycombing (curved arrow), most prominent in the middle and upper lung zones. These changes correlate with the presence of fibrosis on lung biopsy. The upper lung field predominance supports the diagnosis of hypersensitivity pneumonitis.

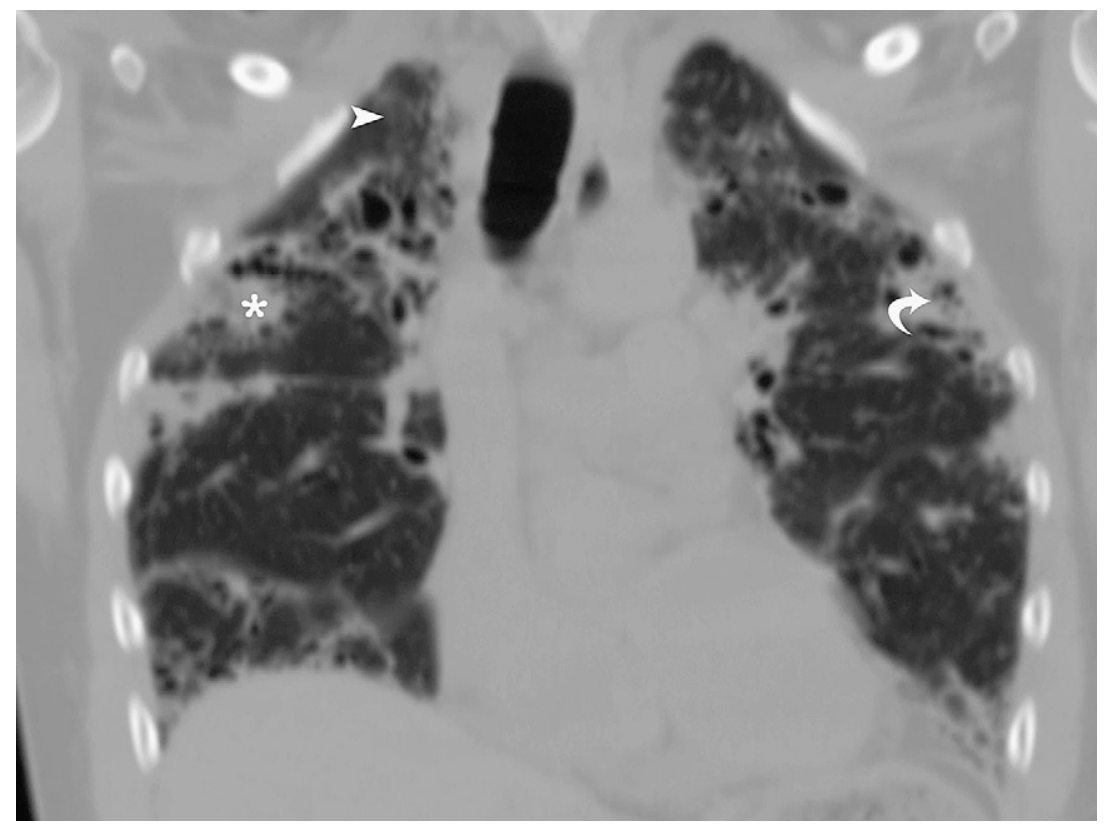




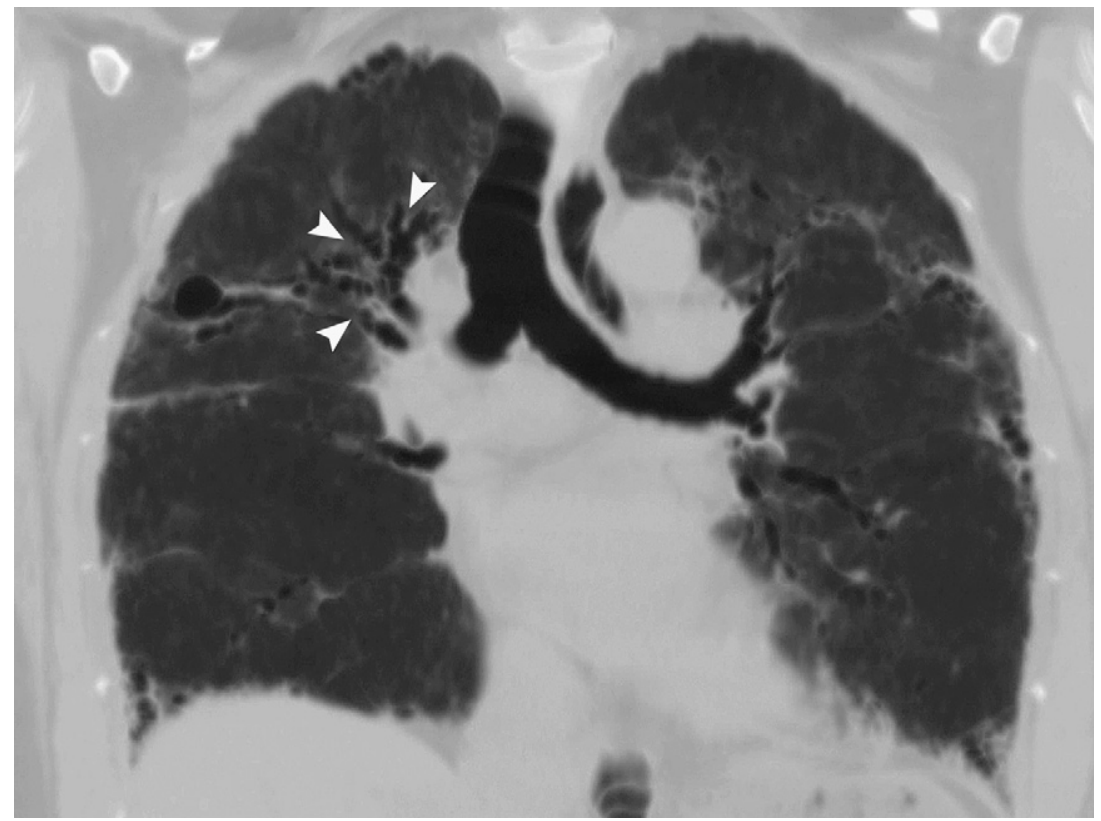

Fig. 5. Hypersensitivity pneumonitis. Coronal reconstruction of highresolution axial $\mathrm{CT}$ data displayed as a minimalintensity pixel projection that allows for detailed airways assessment. There is distortion and dilatation of middle and upper lung zone airways (within area defined by arrowheads), called traction bronchiectasis, which indicates the presence of surrounding fibrosis.

pattern of injury is not evident on transbronchial biopsy.

Histologic findings are the same regardless of etiologic agent. In active disease, the triad of airway-centered (Fig. 9) chronic interstitial inflammation (Fig. 10), poorly circumscribed interstitial non-necrotizing granulomas (Fig. 11), and organizing pneumonia (Fig. 12) is diagnostic of hypersensitivity pneumonitis. All three components, however, are not always present; airway-centered distribution of chronic interstitial inflammation is a constant feature, reflecting the portal of entry of the etiologic agent, but granulomas resolve with time. Additionally, the luminal plugs of organizing pneumonia may be inconspicuous or absent. Chronic interstitial inflammatory infiltrates vary from an airway-centered distribution with little or no involvement of the intervening distal parenchyma to diffuse distribution across the secondary lobule with maintenance of accentuation around airways. Lymphocytes constitute most interstitial infiltrates with plasma cells usually present but in

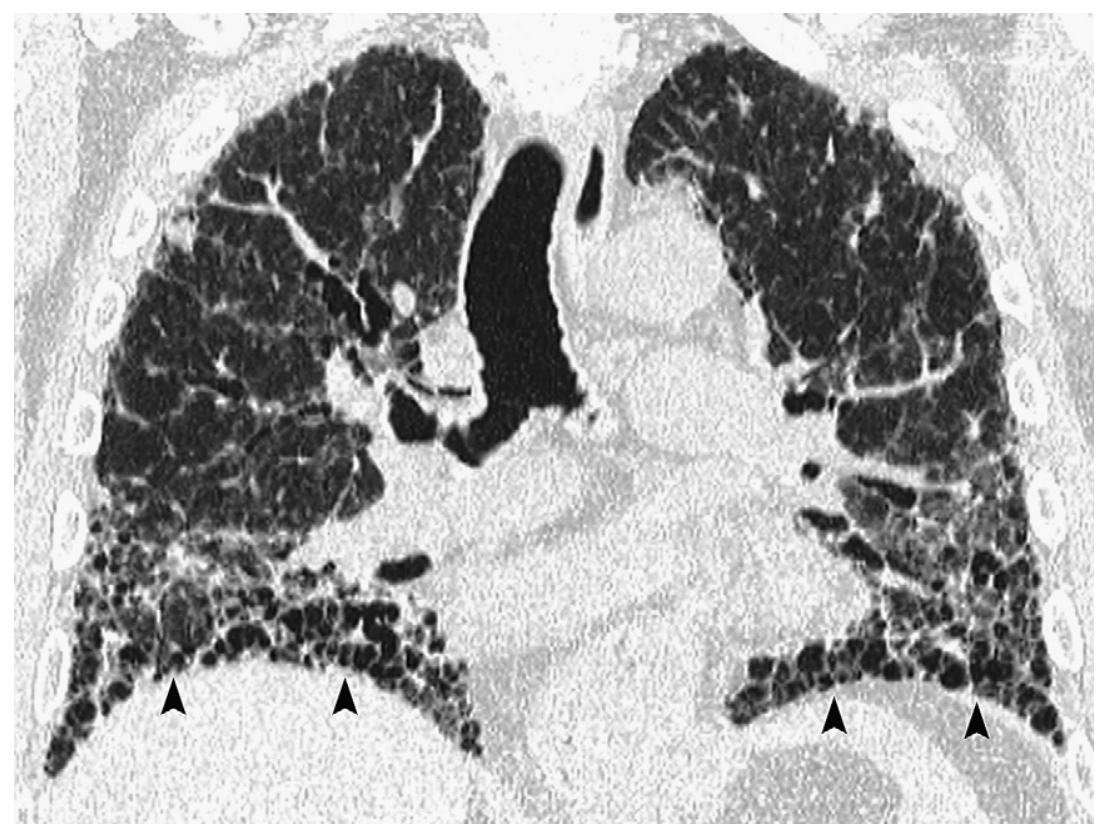

Fig. 6. Idiopathic pulmonary fibrosis. Coronal reconstruction of highresolution axial $\mathrm{CT}$ data demonstrates peripheral reticulation and honeycombing with a striking basal predominance diagnostic of IPF (arrowheads). 
Fig. 7. Active infection with Mycobacterium tuberculosis. Coronal reconstruction of high-resolution axial $C T$ data demonstrates focal nodules (within area defined by arrowheads) in the left upper lobe consistent with an infectious process.

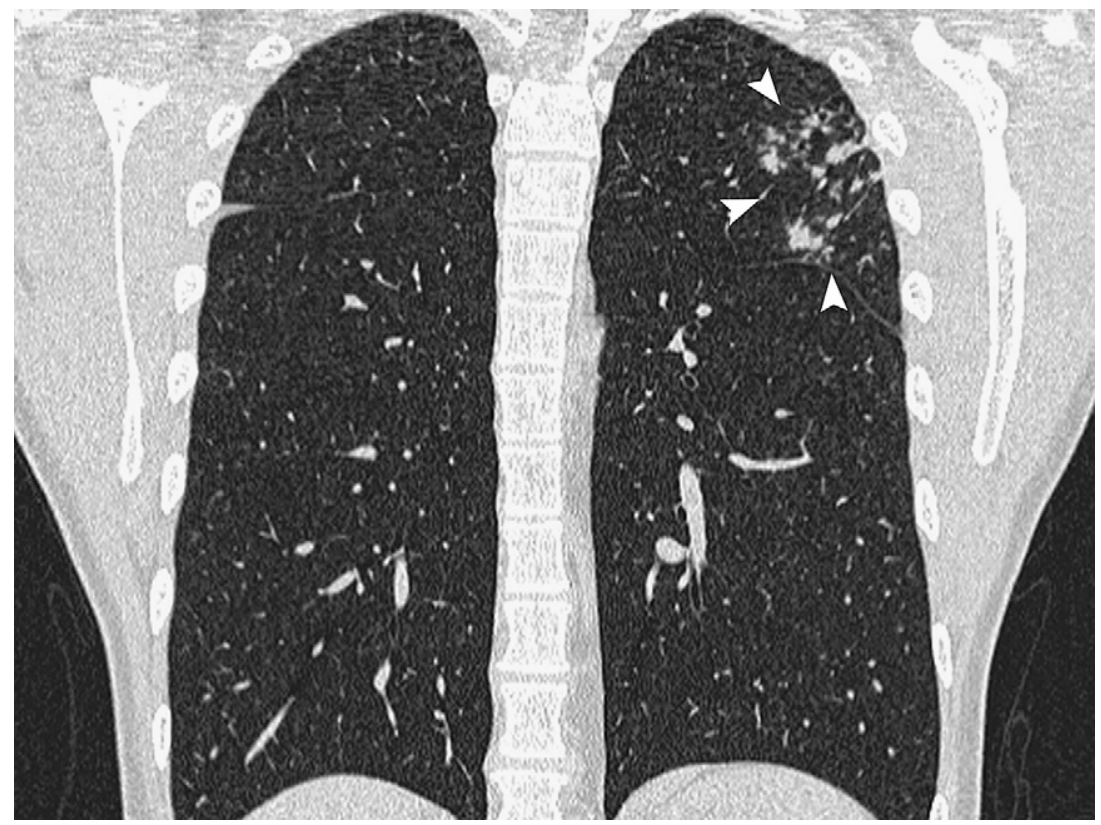

fewer numbers. Granulomas are loose, poorly circumscribed interstitial collections of epithelioid histiocytes admixed with variable numbers of multinucleated giant cells and lymphocytes. Epithelioid histiocytes, separate from poorly formed non-necrotizing granulomas, are often present in the peribronchiolar interstitium. Organizing pneumonia is characterized by plugs of pale staining, loose fibroblastic tissue filling distal bronchioles (also termed bronchiolitis obliterans), alveolar ducts, and contiguous alveolar spaces.
Foamy macrophages are seen in alveolar spaces secondary to bronchiolar obstruction; rarely these are accompanied by a fibrinous exudate and neutrophils. Early on, interstitial fibrosis is minimal, but continued exposure can lead to fibrotic residua in the histologic patterns of fibrotic nonspecific interstitial pneumonia (NSIP) or usual interstitial pneumonia (UIP). ${ }^{17}$ Lymphoid follicles with germinal centers, interstitial eosinophils and neutrophils, and vasculitis are typically absent.
Fig. 8. Mycobacterium intracellulare avium (MAI) infection. Coronal reconstruction of high-resolution axial CT data demonstrates bronchiectasis and associated ground glass in the anterior segment of the right upper lobe (within area defined by arrowheads). The focal anterior location of the disease is highly suggestive of MAI infection.

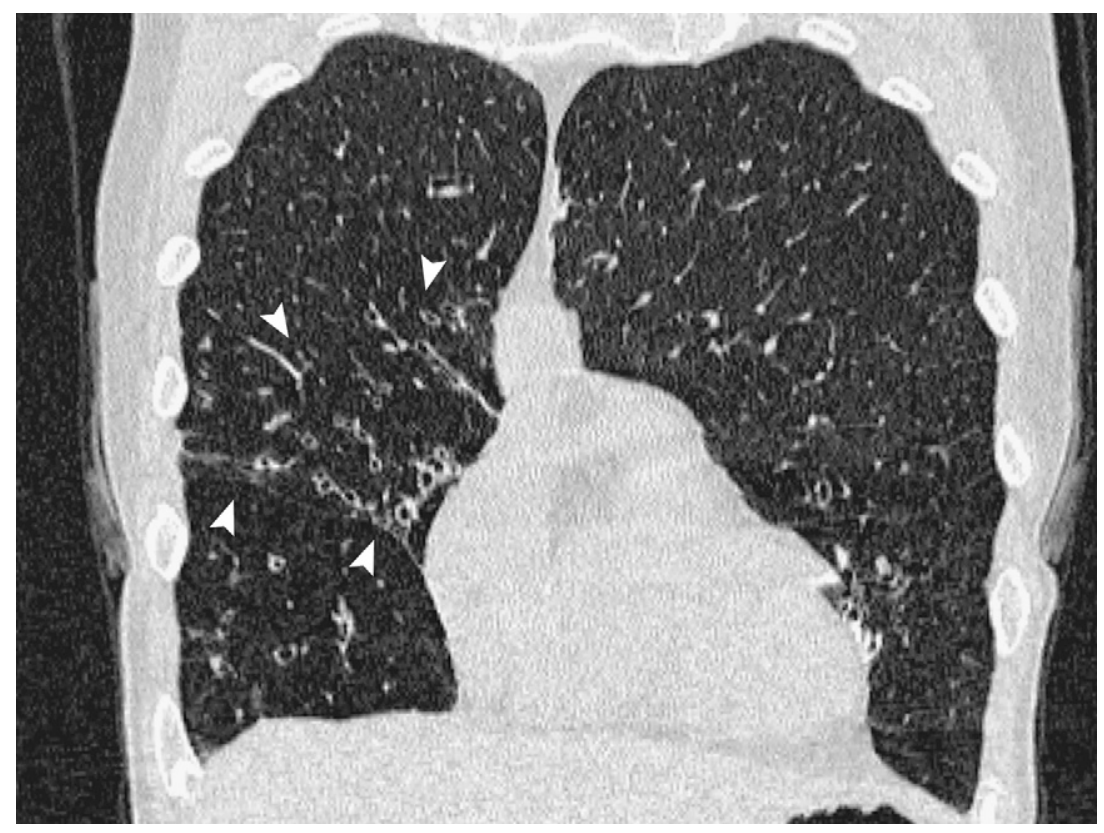




\section{DIFFERENTIAL DIAGNOSIS}

The histologic differential diagnosis of hypersensitivity pneumonitis can be organized into two groups:

1. Noninfectious interstitial lung disease, primarily sarcoidosis, lymphoid interstitial pneumonia (LIP), NSIP, and UIP

2. Granulomatous infection.

Sarcoidosis generally can be separated from hypersensitivity pneumonitis based on morphology and distribution of the granulomas and the degree and distribution of the chronic interstitial inflammation. In sarcoidosis (Fig. 13), granulomas are compact, well circumscribed, sometimes hyalinized, and distributed in a lymphangitic pattern along bronchovascular bundles, pleura, and interlobular septae. In hypersensitivity pneumonitis, however, granulomas (see Fig. 11) are loose and poorly circumscribed; additionally they lack hyalinization and are confined to a peribronchiolar distribution. Chronic interstitial inflammation in sarcoidosis typically accompanies the distribution of granulomas without significant extension into the adjacent parenchyma; in contrast, hypersensitivity pneumonitis demonstrates a greater degree of interstitial inflammation with more extensive involvement of the surrounding parenchyma. Organizing pneumonia is typically not a feature of sarcoidosis. LIP is differentiated from hypersensitivity pneumonitis by its densely cellular interstitial infiltrates of lymphocytes, plasma cells, and histiocytes that markedly expand and distort alveolar walls. LIP diffusely involves the lung parenchyma and lacks airway-centered distribution. Interstitial non-necrotizing granulomas, giant cells, and small clusters epithelioid histiocytes may be present, but more obvious are the lymphoid aggregates containing reactive germinal centers that are common in LIP. NSIP is a nonspecific form of interstitial fibrosis seen in a number of clinical settings including hypersensitivity pneumonitis. ${ }^{17}$ Diffuse distribution of inflammatory infiltrates, rare or a lack of non-necrotizing granulomas, and obvious organizing pneumonia should signal an etiology other than hypersensitivity pneumonitis; however, identification of hypersensitivity pneumonitis as the etiology of fibrotic NSIP is most often not possible on histology alone and requires inclusion of clinical, laboratory, and radiologic findings. In addition to fibrotic NSIP, the fibrotic residua of hypersensitivity pneumonitis may histologically show UIP. Like NSIP, UIP is a histologic pattern of injury associated with several clinical conditions including, most frequently, IPF, but also hypersensitivity pneumonitis. ${ }^{17}$ Fibrosis is upper lobe with patchy involvement of central and peripheral areas in hypersensitivity pneumonitis in contrast to the lower lobe and strikingly peripheral distribution in IPF. Within the secondary lobule, the fibrosis of hypersensitivity pneumonitis is more airway-centered rather than the paraseptal and subpleural distribution of IPF.

\section{Differential Diagnosis Hypersensitivity Pneumonitis}

\section{Sarcoidosis}

Granulomas are compact, well-circumscribed, distributed in a lymphangitic pattern, and are accompanied by mild chronic interstitial inflammation, while in hypersensitivity pneumonitis granulomas are loose, poorly-formed, and distributed primarily around airways, and the relatively greater chronic interstitial inflammation is airway-centered but also variably involves the adjacent parenchyma.

\section{LIP}

In contrast to hypersensitivity pneumonitis, the lymphoplasmacytic interstitial infiltrates in LIP are considerably more cellular; they are diffuse rather than airway-centered, and they markedly expand and distort alveolar walls.

\section{NSIP}

This is a nonspecific form of interstitial fibrosis seen in a variety of clinical settings including hypersensitivity pneumonitis. Diffuse chronic interstitial inflammation, rare or lack of nonnecrotizing granulomas, and prominent organizing pneumonia suggest an etiology other than hypersensitivity pneumonitis for fibrosis.

\section{UIP}

Histologic UIP does not equal a clinical diagnosis of IPF, and like NSIP is associated with a variety of clinical conditions including hypersensitivity pneumonitis. Upper lobe distribution of histologic UIP favors association with hypersensitivity pneumonitis rather than IPF; the latter is lower lobe in distribution.

\section{Granulomatous infection}

Imaging can provide important clues in determining the significance of granulomas; imaging with focal or unilateral abnormalities militates against diffuse lung processes such as hypersensitivity pneumonitis and should prompt a careful search for an infectious etiology. 
Fig. 9. Hypersensitivity pneumonitis. Scanning magnification demonstrates the characteristic airway-centered distribution of lung injury, which reflects the portal of entry of the etiologic agent $(1 \times)$.

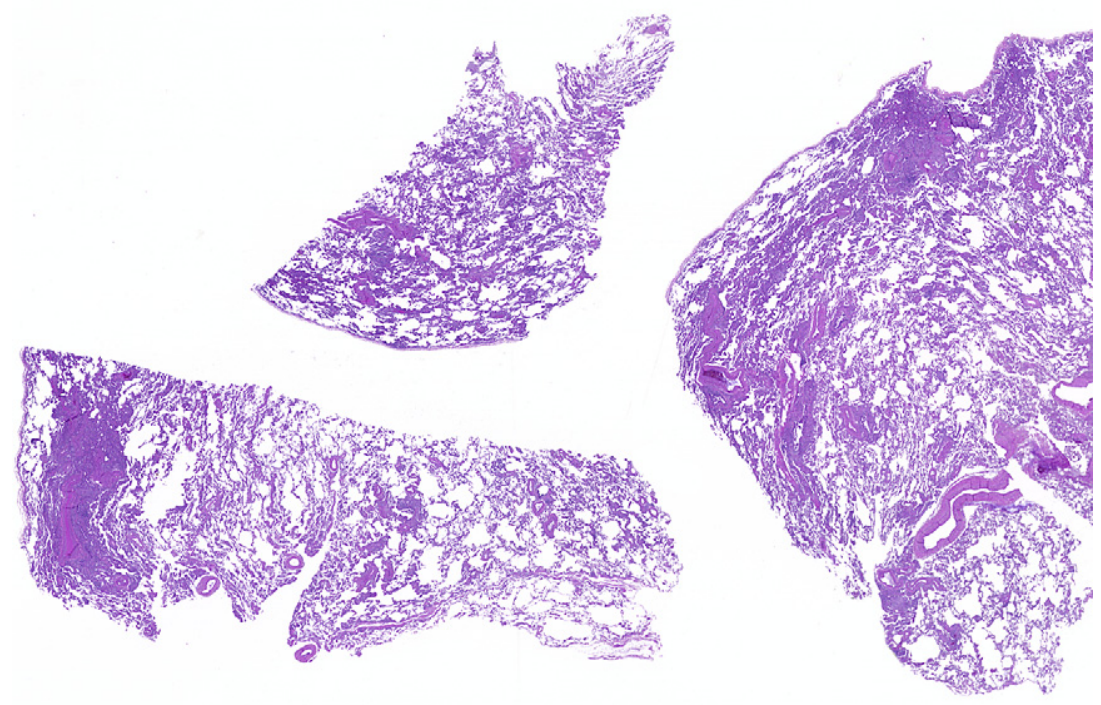

Special stains for microorganisms should be performed in all biopsies with granulomas and correlated with cultures to separate hypersensitivity pneumonitis from granulomatous infections. Although controversial regarding whether it represents a hypersensitivity reaction or infection, hot tub lung often is considered in differential diagnosis of hypersensitivity pneumonitis. ${ }^{18,19}$ Hot tub lung is characterized by airway-centered non-necrotizing granulomas, organizing pneumonia, and chronic interstitial inflammation. In contrast to hypersensitivity pneumonitis, however, the granulomas are well-formed and distributed in both the interstitium and alveolar spaces; organizing pneumonia is more prominent, and the chronic interstitial inflammation tends to be associated with the granulomas rather than diffusely distributed in the lung parenchyma.
Fig. 10. Hypersensitivity pneumonitis. Airway-centered chronic interstitial inflammation comprised primarily of lymphocytes is a constant finding $(100 \times)$.

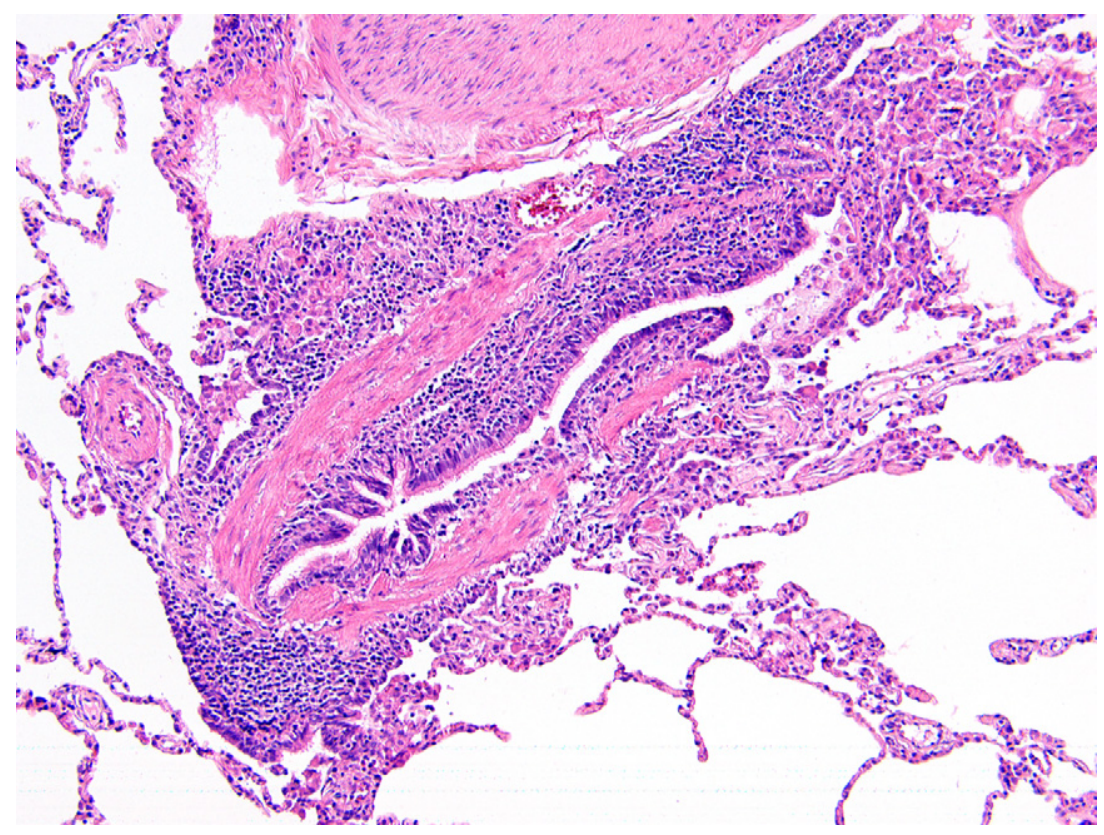




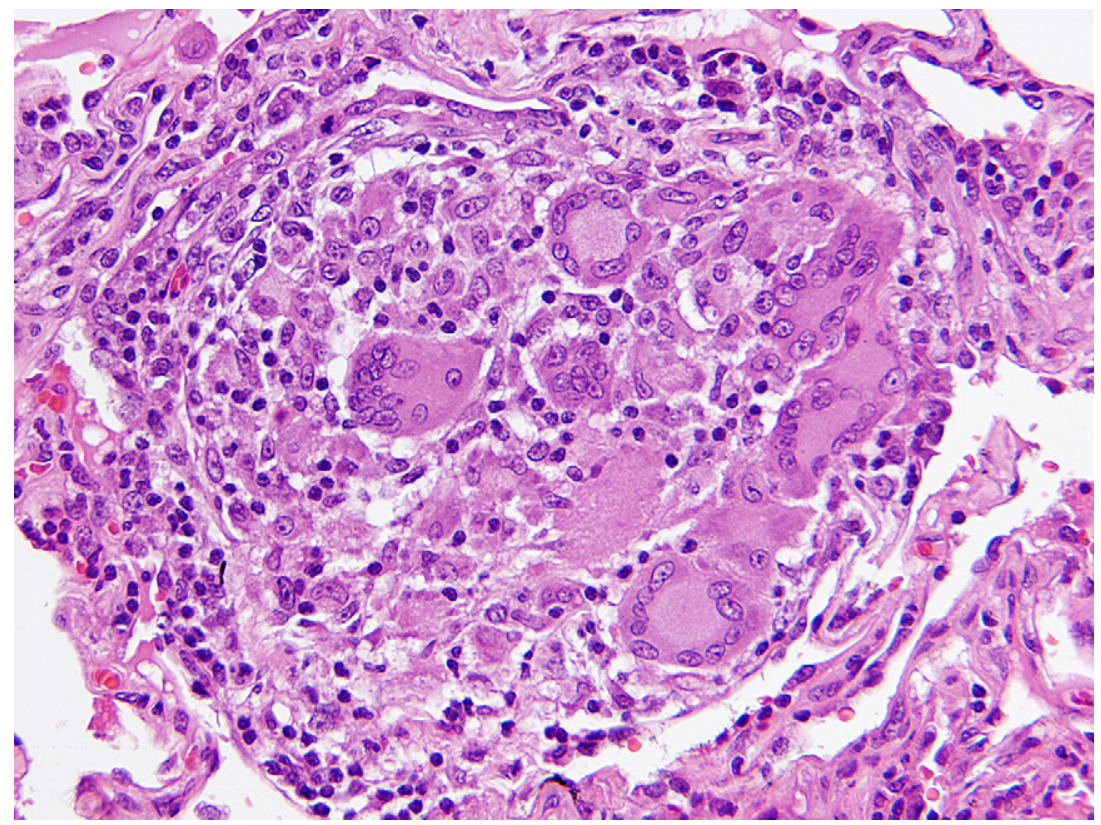

Fig. 11. Hypersensitivity pneumonitis. Granulomas are non-necrotizing, poorly circumscribed interstitial aggregates of epithelioid histiocytes admixed with variable numbers of multinucleated giant cells and lymphocytes $(320 \times)$.

\section{AN APPROACH TO BIOPSY DIAGNOSIS}

Interpretation of biopsies in this setting is difficult since granulomas occur in a wide array of disease processes. The best approach to diagnosis is derived from piecing together the multidisciplinary triangle of clinical, radiologic, and pathologic information. ${ }^{17}$ On a practical basis, this goal is often difficult to achieve; at minimum, the authors review the pathology in conjunction with imaging studies. Depending on the type of biopsy and histologic findings in a given case, pathologists will have a greater or lesser degree of confidence in rendering the diagnosis of hypersensitivity pneumonitis. In combination with the histology, imaging plays a critical role in corroborating the diagnosis, but equally important, imaging can suggest an infectious etiology for granulomatous change on biopsy rather than hypersensitivity pneumonitis.

The greatest degree of confidence occurs in active disease when the triad of airway-centered chronic interstitial inflammation, poorly-formed

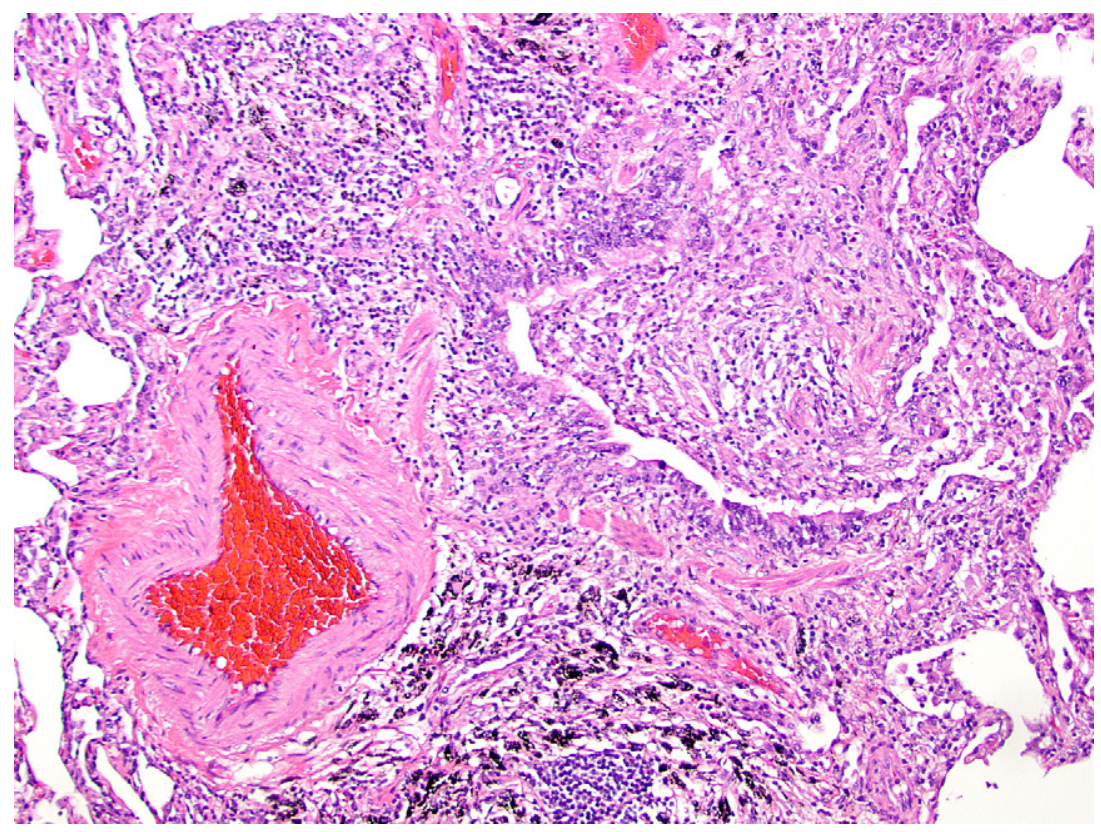

Fig. 12. Hypersensitivity pneumonitis. Organizing pneumonia is characterized by plugs of loose fibroblastic tissue within lumens of bronchioles, alveolar ducts, and adjacent alveolar spaces $(100 \times)$. 
Fig. 13. Sarcoidosis. In contrast to the granulomas in hypersensitivity pneumonitis (see Fig. 11), granulomas in sarcoidosis are compact and well circumscribed, and as seen in this example, they can demonstrate peripheral, concentric layers of hyalinization $(160 \times)$.

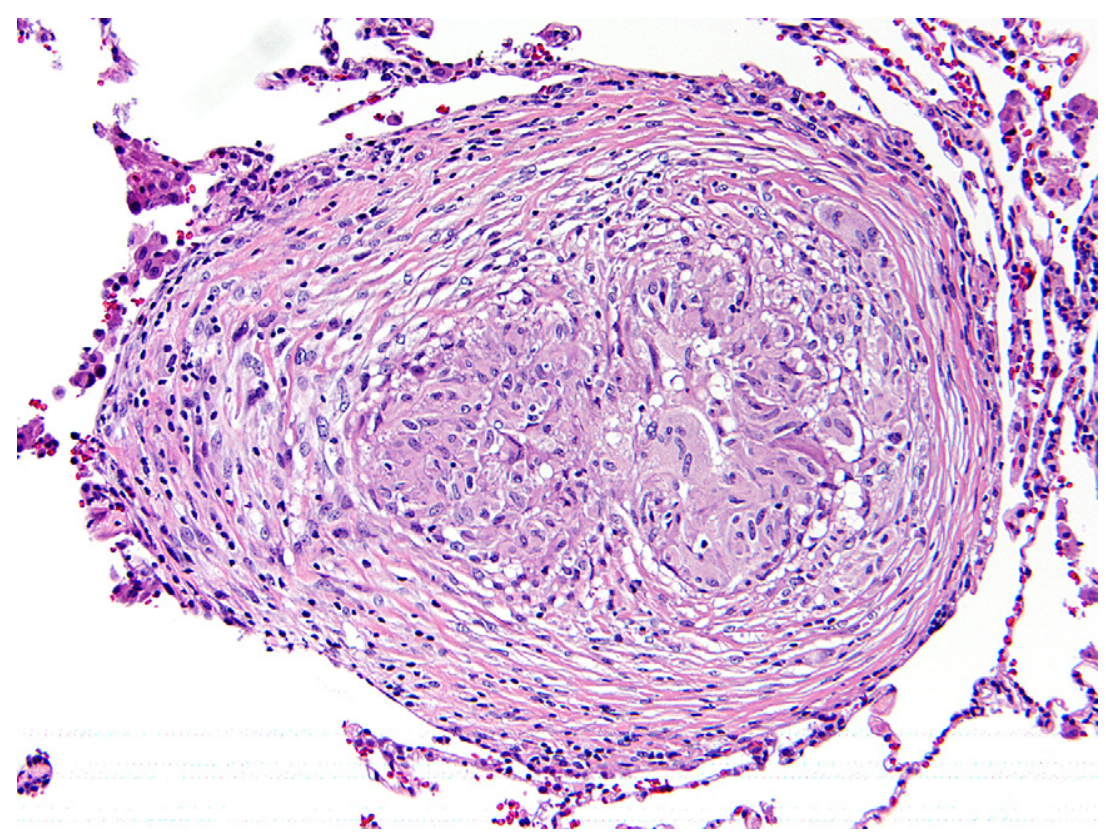

granulomas, and organizing pneumonia is present on biopsy and is coupled with widespread mosaic ground glass attenuation and centrilobular nodules on chest CT. Airway-centered chronic interstitial inflammation with poorly formed granulomas, or solely airway-centered chronic interstitial inflammation coupled with characteristic imaging also would support the diagnosis, although with decreasing levels of confidence. In residual disease when the biopsy demonstrates histologic UIP, the presence of epithelioid histocytes and granulomas should suggest hypersensitivity pneumonitis as the etiology of the fibrosis rather than IPF, since epithelioid histiocytes and granulomas are not features of IPF. Imaging can be extremely helpful in this setting as distribution throughout the lungs is generally more apparent on imaging than on even a substantial surgical lung biopsy; histologic UIP associated with hypersensitivity pneumonitis shows upper and middle lung zone involvement on CT in contrast to histologic UIP that is associated with IPF, which demonstrates lower lobe, strikingly peripheral involvement. When faced with any biopsy, but particularly limited surgical lung biopsies and transbronchial biopsies, imaging can prevent pathologists from making errors in determining the significance of granulomas by demonstrating localized findings compatible with infection. Lung injury caused by infection is more common than injury caused by hypersensitivity pneumonitis, and the chest CT plays a critical role in identifying particularly typical and atypical mycobacterial infections (see Figs. 7 and 8).

\section{PATHOGENESIS, PROGNOSIS, AND}

\section{TREATMENT}

Immune system, host, and exposure-related factors interact to play a role in the pathogenesis of hypersensitivity pneumonitis. Immunologic sensitization from repeated antigen exposure results in immune-mediated lung inflammation. Both immune complex and cellular responses contribute to the inflammatory response with

Pitfalls
HISTOLOGIC EvALUATION OF
HYPERSENSITIVITY PNEUMONITIS
! Interpreting biopsies in the absence of clinical
and radiologic findings; correlation with
imaging to determine distribution of disease
is prudent and can help avoid errors in
diagnosis
! Failing to perform special stains for microor-
ganisms and to correlate with cultures when
granulomas are present on biopsy
! Assuming that negative special stains exclude
infection. The authorshave reviewed a number
of cases in their consultation practice of non-
necrotizing granulomas having negative
special stains that were signed out by the
pathologist as either hypersensitivity pneumo-
nitis or sarcoidosis, while the HRCT demon-
strated typical findings of Lady Windermere
syndrome (a Mycobacterium avium complex
disease) and cultures were confirmatory


high titers of antigen-specific precipitating serum immunoglobulin G ( $\mathrm{lgG}$ ) supporting the involvement by the former, and lymphoid infiltrates and non-necrotizing granulomas heralding involvement by the latter. ${ }^{7,20} \mathrm{BAL}$ fluid shows a lymphocytosis, accounting for $60 \%$ to $80 \%$ of cells recovered, with a predominance of CD8 suppressor/cytotoxic T cells. Host susceptibility is reflected in the observation that many people are exposed to antigens associated with hypersensitivity pneumonitis, but only a small percentage develops disease. Smoking is thought to confer protection as the disease mainly occurs in nonsmokers. ${ }^{21-23}$ Exposure-related factors encompassing type, intensity, and duration of exposure to the offending agent, antigen concentration and solubility, and particle size all influence development of disease. ${ }^{24}$ Avoiding contact with the offending antigen is the cornerstone of hypersensitivity pneumonitis treatment; however, even with cessation the clinical course is variable. $^{2}$ Active disease typically resolves without sequelae. ${ }^{25}$ Oral corticosteroids serve to control symptoms but do not appear to effect long-term outcome. ${ }^{7}$ The presence of fibrosis on histology or CT portents a poor prognosis. ${ }^{13}$

\section{REFERENCES}

1. Lacasse Y, Selman M, Costabel U, et al. Classification of hypersensitivity pneumonitis: a hypothesis. Int Arch Allergy Immunol 2009;149:161-6.

2. Glazer CS, Rose CS, Lynch DA. Clinical and radiologic manifestations of hypersensitivity pneumonitis. J Thorac Imaging 2002;17:261-72.

3. Schuyler M, Cormier Y. The diagnosis of hypersensitivity pneumonitis. Chest 1997;111:534-6.

4. Cormier Y, Israel-Assayag E, Desmeules M, et al. Effect of contact avoidance or treatment with oral prednisolone on bronchoalveolar lavage surfactant protein A levels in subjects with farmer's lung. Thorax 1996;51:1210-5.

5. Richerson HB, Bernstein IL, Fink JN, et al. Guidelines for the clinical evaluation of hypersensitivity pneumonitis. Report of the Subcommittee on Hypersensitivity Pneumonitis. J Allergy Clin Immunol 1989;84:839-44.

6. Terho EO. Diagnostic criteria for farmer's lung disease. Am J Ind Med 1986;10:329.

7. Girard M, Lacasse $Y$, Cormier Y. Hypersensitivity pneumonitis. Allergy 2009;64:322-34.

8. Lacasse Y, Selman M, Costabel U, et al. Clinical diagnosis of hypersensitivity pneumonitis. Am J Respir Crit Care Med 2003;168:952-8.

9. Franks TJ, Galvin JR, Frazier AA. The impact and use of high-resolution computed tomography in diffuse lung disease. Curr Diag Pathol 2004;10:279-90.

10. Lynch DA, Rose CS, Way D, et al. Hypersensitivity pneumonitis: sensitivity of high-resolution CT in a population-based study. Am J Roentgenol 1992; 159:469-72.

11. Hansell DM, Wells AU, Padley SP, et al. Hypersensitivity pneumonitis: correlation of individual CT patterns with functional abnormalities. Radiology 1996;199:123-8.

12. Hirschmann JV, Pipavath SN, Godwin JD. Hypersensitivity pneumonitis: a historical, clinical, and radiologic review. Radiographics 2009;29:1921-38.

13. Hanak V, Golbin JM, Hartman TE, et al. High-resolution CT findings of parenchymal fibrosis correlate with prognosis in hypersensitivity pneumonitis. Chest 2008;134:133-8.

14. Lynch DA, Newell JD, Logan PM, et al. Can CT distinguish hypersensitivity pneumonitis from idiopathic pulmonary fibrosis? Am J Roentgenol 1995;165:807-11.

15. Silva Cl, Muller NL, Lynch DA, et al. Chronic hypersensitivity pneumonitis: differentiation from idiopathic pulmonary fibrosis and nonspecific interstitial pneumonia by using thin-section CT. Radiology 2008;246:288-97.

16. Silva $\mathrm{Cl}$, Churg A, Muller NL. Hypersensitivity pneumonitis: spectrum of high-resolution CT and pathologic findings. Am J Roentgenol 2007;188:334-44.

17. American Thoracic Society, European Respiratory Society. American Thoracic Society/European Respiratory Society International Multidisciplinary Consensus Classification of the Idiopathic Interstitial Pneumonias. This joint statement of the American Thoracic Society (ATS), and the European Respiratory Society (ERS) was adopted by the ATS board of directors, June 2001 and by the ERS Executive Committee, June 2001. Am J Respir Crit Care Med 2002;165:277-304.

18. Agarwal R, Nath A. Hot-tub lung: hypersensitivity to Mycobacterium avium but not hypersensitivity pneumonitis. Respir Med 2006;100:1478.

19. Hanak V, Kalra S, Aksamit TR, et al. Hot tub lung: presenting features and clinical course of 21 patients. Respir Med 2006;100:610-5.

20. Bourke SJ, Dalphin JC, Boyd G, et al. Hypersensitivity pneumonitis: current concepts. Eur Respir J 2001;32:81S-91S.

21. Baldwin Cl, Todd A, Bourke S, et al. Pigeon fanciers' lung: effects of smoking on serum and salivary antibody responses to pigeon antigens. Clin Exp Immunol 1998;113:166-72.

22. Navarro C, Mejia M, Gaxiola M, et al. Hypersensitivity pneumonitis: a broader perspective. Treat Respir Med 2006;5:167-79.

23. Warren CP. Extrinsic allergic alveolitis: a disease commoner in non-smokers. Thorax. 1977;32:567-9.

24. Cordeiro CR, Jones JC, Alfaro T, et al. Bronchoalveolar lavage in occupational lung diseases. Semin Respir Crit Care Med 2007;28:504-13.

25. Cormier Y, Belanger J. Long-term physiologic outcome after acute farmer's lung. Chest 1985;87:796-800. 